(C) 2009 IEEE. Personal use of this material is permitted. Permission from IEEE must be obtained for all other uses, in any current or future media, including reprinting/republishing this material for advertising or promotional purposes, creating new collective works, for resale or redistribution to servers or lists, or reuse of any copyrighted component of this work in other works. 


\title{
MSE Bounds for Phase Estimation in Presence of Recursive Nuisance Parameters
}

\author{
Xiaojing Huang and Y. Jay Guo \\ Wireless Technologies Laboratory \\ CSIRO ICT Centre \\ Sydney, Australia \\ Xiaojing.Huang@csiro.au and Jay.Guo@csiro.au
}

\begin{abstract}
The mean squared error (MSE) is commonly used to measure and compare the performance of various phase estimation techniques in communications and signal processing systems. When the received signal contains recursive nuisance parameters, the MSE is extremely difficult to obtain and even the conventional modified Cramér-Rao bound (MCRB) can not be readily applied. In this paper, a recursive MSE bound and its simplified calculation method are proposed to solve the problem. As an application example, an adaptive hybrid antenna array and its associated angle-of-arrival (AoA) estimation technique are presented. The MSE of the AoA estimation is simulated and compared with the recursive MSE bound and MCRB. The results show that the proposed recursive MSE bound provides a tighter lower MSE bound than the recursive MCRB.
\end{abstract}

Keywords-Phase estimation, mean squared error, Cramér-Rao bound, and modified Cramér-Rao bound.

\section{INTRODUCTION}

Phase estimation is a fundamental technique in communications and signal processing systems [1-4]. Demodulation of phase modulated signals and carrier frequency synchronization are the most common applications of phase estimation. In radar and communications systems employing phased antenna arrays, phase estimation is also essential to detect the angle-of-arrival (AoA) of the incident signal. Based on the AoA information, the phases of the antenna elements can be adaptively adjusted to increase the signal-to-noise ratio (SNR) of the received signal [2,3,11-13].

The mean squared error (MSE) is commonly used to measure and compare the performance of different phase estimation techniques [6,7]. Unfortunately, the MSE is often infeasible to obtain, especially when random nuisance parameters are present in the received signals. This difficulty is somewhat moderated by using the Cramér-Rao bound (CRB) and modified Cramér-Rao bound (MCRB) to give the lower MSE bounds for the estimation and hence provide a meaningful indication of the estimation performance at relatively high SNRs [5-10].

In many applications such as the above mentioned AoA estimation for phased antenna arrays, however, the received signal sometimes contains recursive nuisance parameters $[12,13]$, that is, the previously estimated phases are fed back to the received signal and thus impact on the phase estimation which follows. For phase estimations under this condition, the direct evaluation of the MSE is prohibitively difficult, and even the MCRB can not be readily applied. This is because the MSE evaluation requires the unconditional probability density function (pdf) of a phase estimate, but this pdf at a given time instant can only be determined if the unconditional pdfs of the phase estimates at all previous times are determined first. This recursive nature in finding the unconditional pdf of a phase estimate not only makes the MSE analysis impossible but also the numerical methods extremely difficult. To the authors' knowledge, a better way to overcome the difficulty in analytically evaluating the performance for the phase estimation in presence of recursive nuisance parameters has not been found in the literature.

In this paper, a recursive MSE bound is proposed to evaluate the phase estimation performance in the presence of recursive nuisance parameters using an adaptive hybrid antenna array for millimeter wave (mm-wave) communications [12,13] as the application example. The main contribution is the simplification in obtaining the unconditional pdf of a phase estimate by using a known phase distribution function according to the property of the received information-bearing signal and a recursively determined average SNR of the statistic for the phase estimation. This way, the recursion on pdf is shifted to the recursion on SNR, thus resulting in a recursive MSE bound as well as a recursive MCRB. A closedform expression of the recursive MSE bound is also derived at a given average SNR.

The rest of the paper is organized as follows. In Section II, the signal model for phase estimation in presence of recursive nuisance parameters is described using the hybrid antenna array. In Section III, the infeasibility of the maximumlikelihood (ML) method for the phase estimation is explained. A sub-optimal method is thus proposed and the difficulty in evaluating the MSE performance is discussed. In Section IV, the recursive MSE bound is proposed and a closed-form expression of the bound is given to simplify the calculation. The AoA estimation performance using the hybrid antenna array is also simulated and compared with the analytical results. Finally, conclusions are drawn in Section V.

\section{SIGNAL MODEL}

To show that the problem of phase estimation in presence of recursive nuisance parameters is of practical interest and importance, an application example is given in the following. It relates to the AoA estimation and beamforming for an 
adaptive hybrid antenna array of subarrays used in high data rate ad hoc mm-wave communications systems [12,13], where the recursive MSE bound can be applied to evaluate the AoA estimation performance.

Fig. 1 shows the hybrid antenna array and beamformer structure. For simplicity, only two linear interleaved subarrays are considered. Each linear subarray is composed of $N$ antenna elements and respective analogue phase shifters $\alpha_{0}$, $\alpha_{1}, \ldots, \alpha_{N-1}$. The signals received by the antenna elements in a subarray are combined after phase shifting to produce the subarray signal, and the subarray signal is then converted to digital domain via an analogue-to-digital converter (A/D). The digital subarray signals are denoted as $s_{0}(n)$ and $s_{1}(n)$ respectively. It is seen that the use of analogue subarrays can produce less digital signals for the digital beamformer, and thus significantly reduce the digital signal processing cost, which is an important design consideration for wideband $\mathrm{mm}$ wave communications. The purpose of the AoA estimation is to determine the incident angle of the received informationbearing signal and adjust the analogue phase shifters in the subarrays as well as the digital weights $w_{0}^{*}$ and $w_{1}^{*}$ so that the hybrid antenna array can be beamformed to maximize SNR.

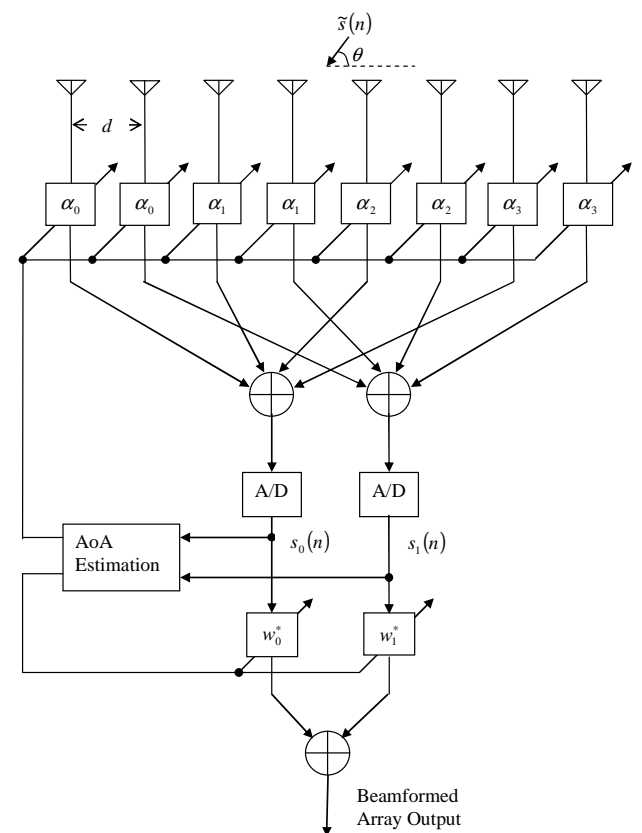

Fig. 1. Adaptive hybrid antenna array and beamformer with two interleaved linear subarrays of size $N=4$.

Assuming that each antenna element has an omni-directional radiation pattern, the received subarray signal is given by

$$
s_{m}(n)=\tilde{s}(n) P_{s}(\theta) \exp \left(j m \frac{2 \pi}{\lambda} d \sin \theta\right)+v_{m}(n), \quad m=0,1,
$$

where $\tilde{s}(n)$ is the information-bearing signal with incident angle $\theta, P_{s}(\theta)=\sum_{i=0}^{N-1} \exp \left\{j\left(i \frac{2 \pi}{\lambda} 2 d \sin \theta+\alpha_{i}\right)\right\}$ is the equivalent array factor of the subarrays, $\lambda$ is the wavelength of the radio frequency signal, $d$ is the element spacing, and $v_{m}(n)$ is the additive white Gaussian noise presented at the output of the $m$ th subarray. Once $\theta$ is estimated, the phase shifts determined by $\alpha_{i}=-i \frac{2 \pi}{\lambda} 2 d \sin \theta, i=0,1, \cdots, N-1$, will be fed back to the phase shifters in the subarrays to steer the hybrid antenna array towards the incident signal beam.

Since the AoA estimation is performed recursively, the subarray signals received at time index $n$ are influenced by the previously determined phase shifts. Defining a new variable $u=\frac{2 \pi}{\lambda} d \sin \theta$, the subarray signal model can be rewritten as

$$
\begin{aligned}
s_{m}(n) & =\tilde{s}(n) \sum_{i=0}^{N-1} \exp \left\{-j 2\left(u^{(n-1)}-u\right) \cdot i\right\} \exp (j m u)+v_{m}(n) \\
& =s(n) P\left(u^{(n-1)}-u\right) \exp (j m u)+v_{m}(n), m=0,1,
\end{aligned}
$$

where $s(n)=N \tilde{s}(n), u^{(n-1)}$ is the estimate of $u$ at time index $n-1$ which is used to determine the phase shifts $\alpha_{i}^{(n-1)}=-i 2 u^{(n-1)}$, and $P(\Delta u)=\frac{\sin N \Delta u}{N \sin \Delta u} \exp \{-j(N-1) \Delta u\}$ is the normalized array factor of the subarrays, the magnitude of which is shown in Fig. 2 for $N=4$. We see that $P(\Delta u)$ is a periodic function of the phase error $\Delta u$ with period $2 \pi$ and satisfies $|P(\Delta u)| \leq 1$ and $|P(0)|=1$.

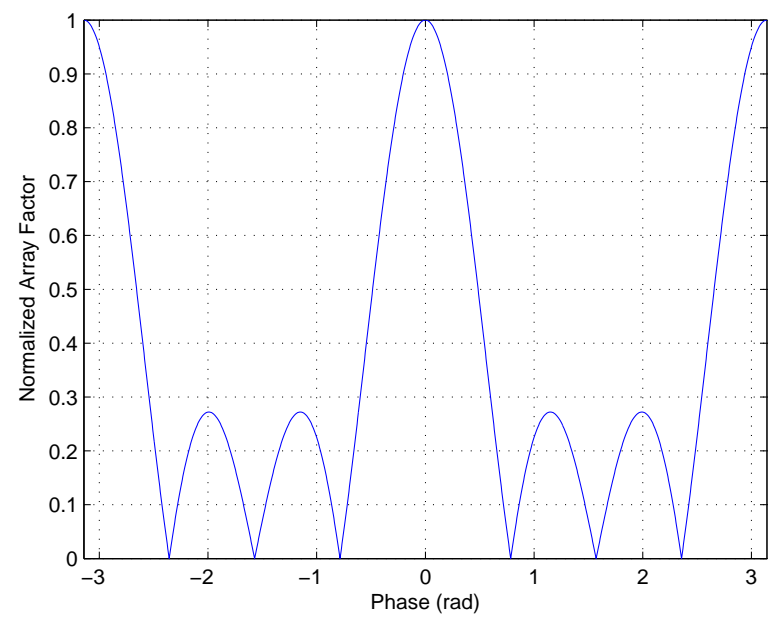

Fig. 2. Magnitude of normalized array factor $P(\Delta u)$.

Assuming that $s(n)$ has average signal power $\sigma_{s}^{2}=E\left\{\left.s(n)\right|^{2}\right\}$ where $E\{\cdot\}$ denotes ensemble expectation and that $v_{0}(n)$ and $v_{1}(n)$ are independent with zero mean and the same noise power $\sigma_{v}^{2}$, the SNR of a subarray signal is defined as $\gamma=\sigma_{s}^{2} / \sigma_{v}^{2}$.

Note that in terms of estimating $u$ at time index $n, s(n)$ 
and $u^{(n-1)}$ are all nuisance parameters. Since $u^{(n-1)}$ is estimated at a previous time, it is called the recursive nuisance parameter. Thus the AoA estimation is formulated as the phase estimation in the presence of recursive nuisance parameters, which is significantly different from the conventional phase estimation models [7-9].

\section{Phase Estimation AND PERFORMANCE}

Given an initial estimate $u^{(0)}$, the ML estimation of $u$ at time index $n$ can be derived as

$$
\begin{aligned}
& u^{(n)}=\arg \min _{\hat{u}}\left\{\sum_{m=0}^{1} \sum_{k=1}^{n}\left|s_{m}(k)-s(k) P\left(u^{(k-1)}-\hat{u}\right) \exp (j m \hat{u})\right|^{2}\right\} \\
&=\arg \max _{\hat{u}}\left\{\operatorname{Re}\left[\sum_{m=0}^{1} \exp (-j m \hat{u}) \sum_{k=1}^{n} s_{m}(k) s^{*}(k) P^{*}\left(u^{(k-1)}-\hat{u}\right)\right]\right. \\
&\left.\left.-\sum_{k=1}^{n} \mid s(k) P\left(u^{(k-1)}-\hat{u}\right)\right)^{2}\right\} .
\end{aligned}
$$

To solve this non-linear optimization problem, a complicated search over $\hat{u}$ in $[-\pi, \pi)$ is required. Moreover, the knowledge of $s(n)$ and perfect synchronization are necessary. Since the subarray SNR is very low before the beam is correctly formed and the signal synchronization is difficult to achieve, the optimal coherent estimation is costly and highly infeasible.

A sub-optimal non-coherent method is therefore preferable using the differential signal between $s_{0}(n)$ and $s_{1}(n)$, i.e.,

$$
r(n)=s_{0}^{*}(n) s_{1}(n)=\left|s(n) P\left(u^{(n-1)}-u\right)\right|^{2} \exp (j u)+z(n)
$$

where $z(n) \approx s(n) P\left(u^{(n-1)}-u\right) \exp (j u) v_{0}^{*}(n)+s^{*}(n) P^{*}\left(u^{(n-1)}-u\right) v_{1}(n)$ can be approximated as a complex Gaussian variable with zero mean and variance $2 s(n) P\left(u^{(n-1)}-u\right)^{2} \sigma_{v}^{2}$. The estimate is then $u^{(n)}=\arg \left\{R^{(n)}\right\}$ where

$$
R^{(n)}=\sum_{k=1}^{n} r(k)=\sum_{k=1}^{n}\left|s(k) P\left(u^{(k-1)}-u\right)\right|^{2} \exp (j u)+\sum_{k=1}^{n} z(k)
$$

is an approximately Gaussian distributed statistic with the joint conditional pdf of its real and imaginary parts, given $s(k)$ for $k=1,2, \cdots, n$, denoted as $\mathbf{s}$, and $u^{(k-1)}$ for $k=1,2, \cdots, n$, denoted as $\mathbf{u}$

$$
\begin{aligned}
& p_{R^{(n)} \mid \mathbf{s} \mathbf{u}}\left(R_{r}^{(n)}, R_{i}^{(n)} \mid \mathbf{s}, \mathbf{u}\right) \\
& =\frac{1}{2 \pi \sigma_{R^{(n)}}^{2}} \exp \left(-\frac{\left(R_{r}^{(n)}-\operatorname{Re}\left\{m_{R^{(n)}}\right\}\right)^{2}+\left(R_{i}^{(n)}-\operatorname{Im}\left\{m_{R^{(n)}}\right\}\right)^{2}}{2 \sigma_{R^{(n)}}^{2}}\right)
\end{aligned}
$$

where $R_{r}^{(n)}$ and $R_{i}^{(n)}$ are the real and imaginary parts of $R^{(n)}$ respectively, $m_{R^{(n)}}=\left.\sum_{k=1}^{n} s(k) P\left(u^{(k-1)}-u\right)\right|^{2} \exp (j u)$ is the conditional mean of $R^{(n)}, \sigma_{R^{(n)}}^{2}=\sum_{k=1}^{n}\left|s(k) P\left(u^{(k-1)}-u\right)\right|^{2} \sigma_{v}^{2}$ is the conditional variance of the real or imaginary part of $R^{(n)}$.
Since $u^{(n)}$ is the phase of a non-zero mean Gaussian distributed signal $R^{(n)}$, the conditional pdf of $u^{(n)}$ is thus [7]

$$
p_{u^{(n)} \mid \mathbf{s}, \mathbf{u}}\left(u^{(n)} \mid \mathbf{s}, \mathbf{u}\right)=p_{0}\left(u^{(n)}-u, \gamma_{\mathbf{s}, \mathbf{u}}^{(n)}\right)
$$

where

$$
p_{0}(x, \gamma)=\frac{1}{2 \pi} e^{-\gamma}\left[1+\sqrt{4 \pi \gamma} \cos x \cdot e^{\gamma \cos ^{2} x} Q(-\sqrt{2 \gamma} \cos x)\right]
$$

which is a periodic function of $x$ with period $2 \pi$, $\gamma_{\mathbf{s}, \mathbf{u}}^{(n)}=\left|m_{R^{(n)}}\right|^{2} / 2 \sigma_{R^{(n)}}^{2}=\frac{1}{2 \sigma_{v}^{2}} \sum_{k=1}^{n}\left|s(k) P\left(u^{(k-1)}-u\right)\right|^{2}$ is the conditional SNR of $R^{(n)}$, and $Q(x)=\frac{1}{2 \pi} \int_{x}^{\infty} e^{-\frac{t^{2}}{2}} d t$ is the Q-function. Eq. (7) indicates that the conditional pdf of $u^{(n)}$ is determined by the conditional SNR of $R^{(n)}$.

The MSE is usually used to measure the performance of the phase estimation. It can be expressed at time index $n$ as

$$
\sigma_{u^{(n)}}^{2}=\int_{-\pi}^{\pi}\left[u^{(n)}-u\right]_{[-\pi, \pi)}^{2} p_{u^{(n)}}\left(u^{(n)}\right) d u^{(n)}
$$

where

$$
p_{u^{(n)}}\left(u^{(n)}\right)=E_{\mathbf{s}, \mathbf{u}}\left\{p_{u^{(n)} \mid \mathbf{s}, \mathbf{u}}\left(u^{(n)} \mid \mathbf{s}, \mathbf{u}\right)\right\}=E_{\mathbf{s}, \mathbf{u}}\left\{p_{0}\left(u^{(n)}-u, \gamma_{\mathbf{s}, \mathbf{u}}^{(n)}\right)\right\}
$$

is the unconditional pdf of $u^{(n)}$ and $[\cdot]_{[-\pi, \pi)}$ means that the argument must be restrained to the interval $[-\pi, \pi)$.

The direct and exact evaluation of MSE from (9) is practically infeasible, because the unconditional pdf of $u^{(n)}$ not only requires the expectation over $s(k)$ for $k=1,2, \cdots, n$, but also the expectation over $u^{(k-1)}$ for $k=1,2, \cdots, n$, which in turn requires the knowledge of the unconditional pdf of $u^{(k-1)}$ for $k=1,2, \cdots, n$. We see that the unconditional pdf of $u^{(n)}$ have to be recursively obtained, which is extremely difficult even with powerful numerical methods.

In presence of nuisance parameters, the difficulty of evaluating the MSE of the phase estimation can be somewhat moderated by alternatively calculating the modified CramérRao bound (MCRB) [8], which gives a lower MSE bound at high SNRs. From (5), the MCRB can be obtained as

$$
\begin{aligned}
\operatorname{MCRB}\left(\sigma_{u^{(n)}}^{2}\right) & =\frac{1}{E_{\mathbf{s}, \mathbf{u}}\left\{E_{R^{(n)} \mid \mathbf{s}, \mathbf{u}}\left\{-\frac{\partial^{2} \ln p_{R^{(n)} \mid \mathbf{s} \mathbf{u}}\left(R_{r}^{(n)}, R_{i}^{(n)} \mid \mathbf{s}, \mathbf{u}\right)}{\partial u^{2}}\right\}\right\}} \\
& =1 / E_{\mathbf{s}, \mathbf{u}}\left\{2 \gamma_{\mathbf{s}, \mathbf{u}}^{(n)}\right\}=1 / 2 \bar{\gamma}^{(n)}
\end{aligned}
$$

where

$$
\begin{aligned}
\bar{\gamma}^{(n)} & =E_{\mathbf{s}, \mathbf{u}}\left\{\gamma_{\mathbf{s}, \mathbf{u}}^{(n)}\right\}=\sum_{k=1}^{n} \frac{E\left\{\left.s(k)\right|^{2}\right\}}{2 \sigma_{v}^{2}} E\left\{\left.P\left(u^{(k-1)}-u\right)\right|^{2}\right\} \\
& =\gamma \sum_{k=1}^{n} E\left\{P\left(u^{(k-1)}-u\right)^{2}\right\}
\end{aligned}
$$

is the average SNR of $R^{(n)}$. It is seen from (11) that even the MCRB is still infeasible to calculated since it also requires the 
unconditional pdf of $u^{(k-1)}$ for $k=1,2, \cdots, n$ to perform the expectation $\left.E\left\{P\left(u^{(k-1)}-u\right)\right)^{2}\right\}$.

\section{RECURSIVE MSE BOUND}

To solve the problem encountered in evaluating the MSE as well as the MCRB, we now replace the unconditional pdf of $u^{(n)}$ expressed in (9) with a known phase distribution determined only by $\bar{\gamma}^{(n)}$ defined in (12). Assuming that $s(n)$ is Gaussian distributed and thus $|s(n)|$ is Rayleigh distributed, the known phase distribution can be chosen as the phase distribution function under Rayleigh fading [7], i.e.,

$$
p_{u^{(n)}}\left(u^{(n)}\right)=p_{1}\left(u^{(n)}-u, \bar{\gamma}^{(n)}\right)
$$

where

$$
p_{1}(x, \gamma)=\frac{1}{2 \pi\left(1+\gamma \sin ^{2} x\right)}+\frac{\sqrt{\gamma} \cos x}{2 \pi\left(1+\gamma \sin ^{2} x\right)^{3 / 2}}\left(\frac{\pi}{2}+\tan ^{-1} \frac{\sqrt{\gamma} \cos x}{\sqrt{1+\gamma \sin ^{2} x}}\right)
$$

which is also a periodic function of $x$ with period $2 \pi$.

To justify the above selection, we simply let $P(\Delta u)=1$ for any $\Delta u$, i.e., there is no recursive nuisance parameter, then $p_{1}\left(u^{(n)}-u, \bar{\gamma}^{(n)}\right)$ will be the true phase pdf in Rayleigh fading channels.

Since $|P(\Delta u)| \leq 1$ when there exists recursive nuisance parameter, which leads to a reduced average SNR of $R^{(n)}$, the actual MSE expressed in (9) will be always larger than that calculated using $p_{1}\left(u^{(n)}-u, \bar{\gamma}^{(n)}\right)$, i.e.,

$$
\begin{aligned}
\sigma_{u^{(n)}}^{2} & \geq \int_{-\pi}^{\pi}\left[u^{(n)}-u\right]_{[-\pi, \pi)}^{2} p_{1}\left(u^{(n)}-u, \bar{\gamma}^{(n)}\right) d u^{(n)} \\
& =\int_{-\pi}^{\pi}\left(u^{(n)}\right)^{2} p_{1}\left(u^{(n)}, \bar{\gamma}^{(n)}\right) d u^{(n)}=\operatorname{MSEB}\left(\sigma_{u^{(n)}}^{2}\right) .
\end{aligned}
$$

Therefore, the right-hand side of (15) represents a lower MSE bound, denoted as $\operatorname{MSEB}\left(\sigma_{u^{(n)}}^{2}\right)$.

Furthermore, from (12) and using the assumption that $P(\Delta u)$ is a periodic function, the initial average SNR can be determined as

$$
\bar{\gamma}^{(1)}=\gamma E\left\{\left.P\left(u^{(0)}-u\right)\right|^{2}\right\}=\frac{1}{2 \pi} \gamma \int_{-\pi}^{\pi}\left|P\left(u^{(0)}\right)\right|^{2} d u^{(0)}
$$

where the initial estimate $u^{(0)}$ is assumed to be uniformly distributed in $[-\pi, \pi)$, and the average SNR for $n>1$ can be recursively determined as

$$
\bar{\gamma}^{(n)}=\bar{\gamma}^{(n-1)}+\gamma \int_{-\pi}^{\pi}\left|P\left(u^{(n-1)}\right)\right|^{2} p_{1}\left(u^{(n-1)}, \bar{\gamma}^{(n-1)}\right) d u^{(n-1)} .
$$

The MSE bound calculated based on the above recursively determined average SNR is thus called recursive MSE bound. With the recursive MSE bound, the difficulty in the recursion on the unconditional pdf of $u^{(n)}$ is simplified to the recursion on the average SNR of $R^{(n)}$. The MCRB based on this average $\mathrm{SNR}$ is called recursive MCRB accordingly.

Further simplification is possible at higher SNR, since $p_{0}(x, \gamma)$ can be approximated as the Gaussian distribution

$$
p_{0}(x, \gamma)=\sqrt{\frac{\gamma}{\pi}} e^{-\gamma x^{2}}, \text { for }-\pi \leq x<\pi,
$$

and accordingly $p_{1}(x, \gamma)$ can be approximated as

$$
p_{1}(x, \gamma)=\frac{\sqrt{\gamma \pi^{2}+1}}{2 \pi\left(\gamma x^{2}+1\right)^{3 / 2}}, \text { for }-\pi \leq x<\pi,
$$

after normalizing it to satisfy the condition $\int_{-\pi}^{\pi} p_{1}(x, \gamma) d x=1$.

Fig. 3 and Fig. 4 show the comparisons of the approximated $p_{0}(x, \gamma)$ and $p_{1}(x, \gamma)$ with their respective true phase pdfs under different $\gamma$ values.

With the above approximation, the recursive MSE bound can be expressed in closed-form as

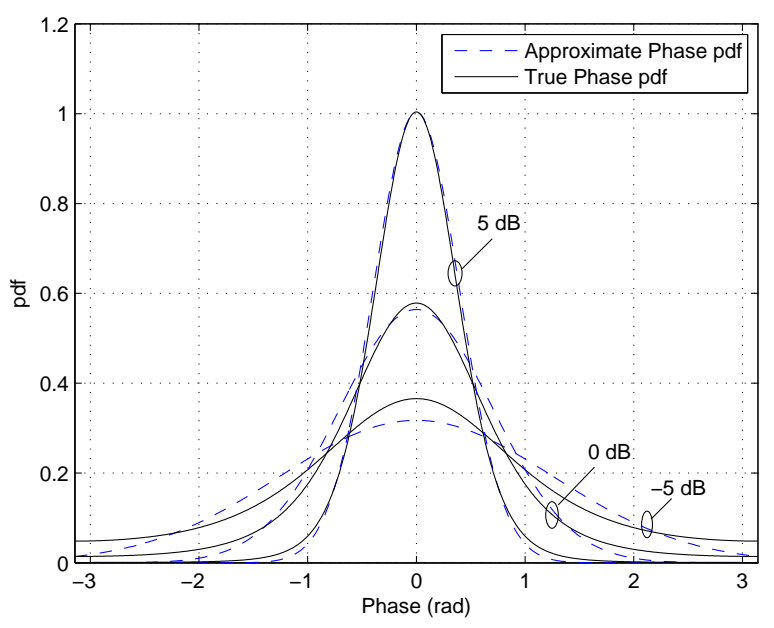

Fig. 3. Approximate and true phase pdfs in Gaussian channel.

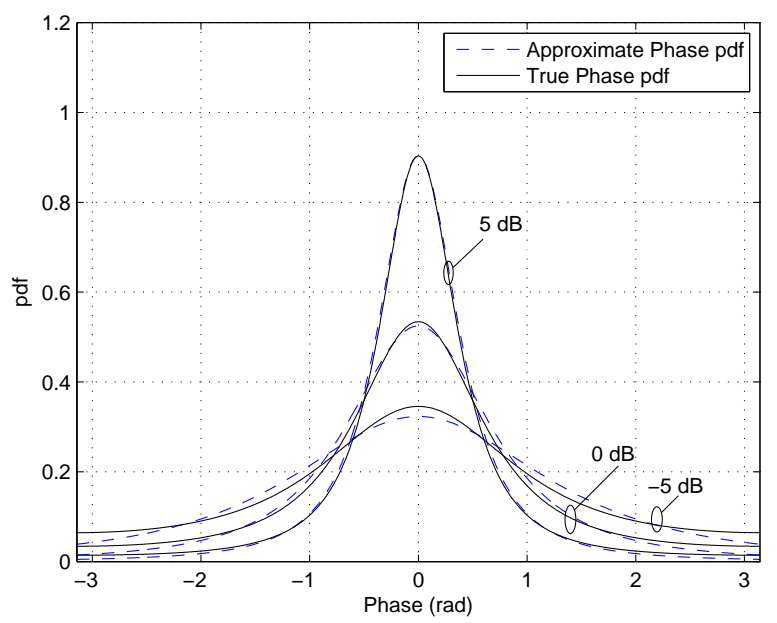

Fig. 4. Approximate and true phase pdfs in Rayleigh fading channel. 


$$
\begin{aligned}
\operatorname{MSEB}\left(\sigma_{u^{(n)}}^{2}\right) & =\int_{-\pi}^{\pi}\left(u^{(n)}\right)^{2} \frac{\sqrt{\bar{\gamma}^{(n)} \pi^{2}+1}}{2 \pi\left[\bar{\gamma}^{(n)}\left(u^{(n)}\right)^{2}+1\right]} d u^{(n)} \\
& =\frac{\sqrt{\bar{\gamma}^{(n)} \pi^{2}+1}}{\pi\left(\bar{\gamma}^{(n)}\right)^{3 / 2}} \sinh ^{-1}\left(\sqrt{\bar{\gamma}^{(n)}} \pi\right)-\frac{1}{\bar{\gamma}^{(n)}}
\end{aligned}
$$

where $\sinh ^{-1}(x)=\ln \left(x+\sqrt{x^{2}+1}\right)$ denotes the inverse hyperbolic sine function.

Simulation of the AoA estimation is performed using the hybrid antenna array shown in Fig. 1 under subarray SNR $\gamma=$ $-2,3$, and $8 \mathrm{~dB}$ respectively. The incident angle is assumed to be $\theta=0$ degree. Fig. 5 shows the estimated $u^{(n)}$ values for one realization under different SNRs, and Fig. 6 shows the simulated MSEs averaged over 1000 realizations. The recursive MSE bounds and the recursive MCRBs are obtained analytically using (20) and (11) respectively based on the recursively determined average SNR calculated by (16) and (17). It is seen that the recursive MSE bound is rather loose at lower SNRs but becomes tighter at higher SNRs. In both cases, the recursive MSE bound provides better performance indication than the recursive MCRB.

\section{CONCLUSIONS}

It has been shown that the true MSE of the phase estimation in presence of recursive nuisance parameters is extremely difficult to obtain and the conventional modified Cramér-Rao bond can not be readily applied. By replacing the unconditional probability density function of a phase estimate with a known phase distribution function according to the statistical property of the information-bearing signal and the recursively obtained average SNR of the statistic for the phase estimation, a recursive MSE bound and its simplified calculation method are derived. The usefulness of the recursive MSE bound is demonstrated through the AoA estimation for an adaptive hybrid antenna array of interleaved subarrays. Simulation results show that the recursive MSE bound provides a tighter MSE lower bound than the recursive modified Cramér-Rao bound.

\section{REFERENCES}

[1] H. Meyr, M. Moeneclaey, and S. Fechtel, Digital Communication Receivers: Synchronization, Channel Estimation, and Signal Processing, New York: Wiley, 1997.

[2] F. B. Gross, Smart Antennas for Wireless Communications, McGrawHill, 2005.

[3] D. Rogstad, A. Mileant, and T. Pham, Antenna Arraying Techniques in the Deep Space Network, Wiley-IEEE, 2003.

[4] J. G. Proakis, Digital Communications, Third Edition, McGraw-Hill, 1995.

[5] S. Bay, C. Herzet, J.-M. Brossier, J.-P. Barbot, and B. Geller, "Analytic and Asymptotic Analysis of Bayesian Cramér-Rao Bound for Dynamical Phase Offset Estimation," IEEE Transactions on Signal Processing, Vol. 56, No. 1, January 2008, pp. 61-70.

[6] A. Papoulis and S. U. Pillai, Probability, Random Variables and Stochastic Processes, Fourth Edition, McGraw-Hill, 2002.

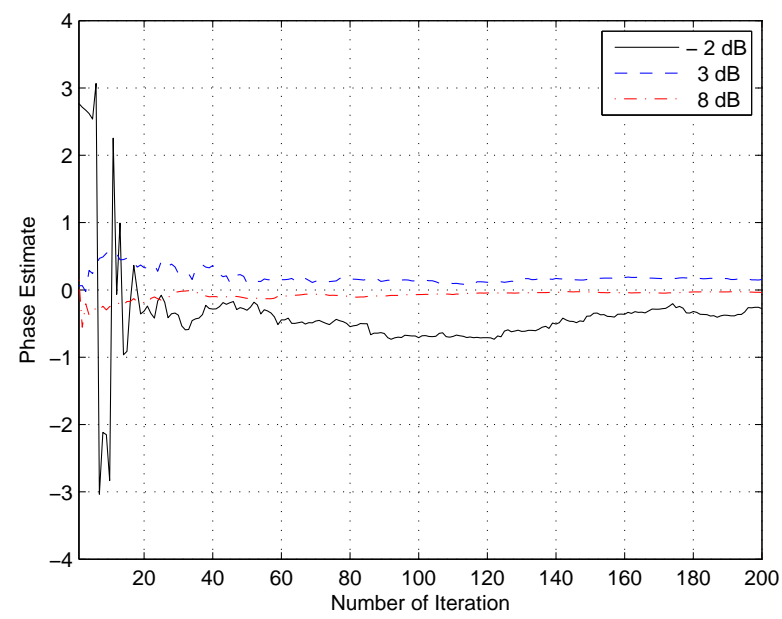

Fig. 5. Estimated phase realizations under different subarray SNRs.

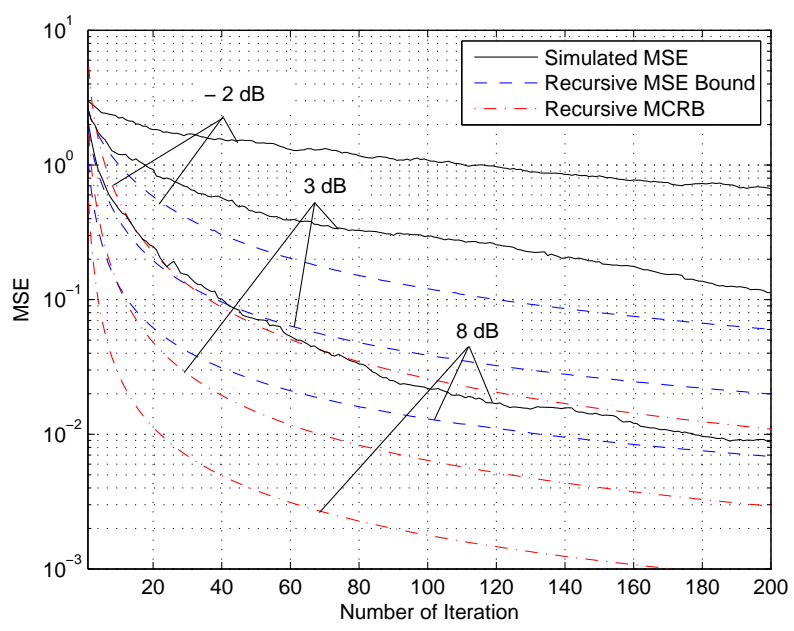

Fig. 6. Simulated MSE vs. recursive MSE bound and recursive MCRB.

[7] R. Reggiannini, "A Fundamental Lower Bound to the Performance of Phase Estimators Over Rician-Fading Channels," IEEE Transactions on Communications, Vol. 45, No. 7, July 1997, pp. 775-778.

[8] A. N. D'Andrea, U. Mengali, and R. Reggiannini, "The Modified Cramér-Rao Bound and Its Application to Synchronization Problems," IEEE Transactions on Communications, Vol. 42, No. 2/3/4, February /March/April 1994, pp. 1391-1399.

[9] F. Gini, R. Reggiannini, and U. Mengali, "The Modified Cramér-Rao Bound in Vector Parameter Estimation," IEEE Transactions on Communications, Vol. 46, No. 1, January 1998, pp. 52-160.

[10] M. Moeneclaey, "On the True and the Modified Cramér-Rao Bounds for the Estimation of a Scalar Parameter in the Presence of Nuisance Parameters," IEEE Transactions on Communications, Vol. 46, No. 11, November 1998, pp. 1536-1544.

[11] Y. Jay Guo, Advances in Mobile Radio Access Networks, Artech House, Inc., 2004.

[12] Y. Jay Guo, J. Bunton, V. Dyadyuk and X. Huang, "Hybrid Adaptive Antenna Array," Australian provisional patent, 2009900371, February 2009.

[13] X. Huang, Y. Jay Guo, and J. Bunton, "Adaptive AoA Estimation and Beamforming with Hybrid Antenna Array of Subarrays," presented at the IEEE Vehicular Technologies Conference (VTC 2009-Fall), Anchorage, USA, 20-23 September 2009. 